\title{
Estudio de los niveles plasmáticos de homocisteína, ácido fólico y vitamina B-12 en una población limeña de jóvenes adultos
}

\author{
MERCEDES SOBERÓN ${ }^{1,3}$, ADA CHARAJA $^{1,3}$, YSELA AGÜERO $^{2,3}$, ROSA ORIONDO $^{1,3}$, \\ MIGUEL SANDOVAL ${ }^{1,3}$, MARCO NÚÑ̃ZZ1,3 \\ ${ }^{1}$ Centro de Investigación de Bioquímica y Nutrición. ${ }^{2}$ Instituto de Investigación de Ciencias Matemáticas. \\ ${ }^{3}$ Universidad Nacional Mayor de San Marcos.
}

\begin{abstract}
Resumen
Objetivo: Estudiar la distribución de homocisteína plasmática y su relación con los niveles de ácido fólico y vitamina B-12, en una población de jóvenes adultos de la ciudad de Lima. Material y Métodos: Estudio de corte transversal en una muestra de 65 personas con edades entre 18 y 30 años. La homocisteína plasmática fue determinada por inmunoensayo de polarización fluorescente (FPIA), ácido fólico por captura iónica y B-12 enzimáticamente. Resultados: La distribución de los niveles de homocisteina varió entre 1,40 y 24,04 mmoles/L, con una media de 8,32 +5,46; mediana 7,76 mmol/L. Los niveles medios de homocisteína fueron significativamente mayores $(p=0,005)$ en varones $(9,97 \pm 4,81 \mathrm{mmol} / \mathrm{L})$, que en mujeres $(7,35 \pm 2,03 \mathrm{mmol} / \mathrm{L})$. Los niveles de homocisteína se correlacionan significativamente con las mediciones de ácido fólico $(\mathrm{r}=-0,329 ; p=0,007)$, pero no hay indicios de asociación con la vitamina B-12; sin embargo, se encontró asociación con el índice de masa corporal (IMC) $(\mathrm{r}=0,391 ; p=0,001)$. Conclusiones: La distribución de los niveles de homocisteína en la muestra estudiada se encuentra dentro de los niveles referenciales sugeridos por la literatura; sin embargo, estos valores tienden a estar próximos al límite inferior del rango de normalidad. Los niveles de ácido fólico en sangre pueden ser un factor determinante de la variación de los niveles de homocisteína.
\end{abstract}

Palabras clave: Homocisteína; ácido fólico; vitamina B-12; inmunoensayo de polarización fluorescente.

Distribution of plasma homocysteine, folate and B-12 vitamin in Lima, Peru's young adults Abstract

Objective: To describe the distribution of plasma homocysteine, folic acid, B-12 vitamin concentrations among students living in the city of Lima, Peru. Material and Methods: Transversal cohort study of 65 young adults between 18 and 30 year-old. The plasma homocysteine concentration was determined by fluorescence polarization inmunoassay FPIA from Abbott Laboratories, folic acid by ionic capture and vitamin B-12 by enzimatic assay. Results: The distribution of homocysteine values ranged from 1,40 to 24,04 mmol /L; geometric mean homocysteine $8,32 \pm 5,46$, median 7,76 mmol/L. Homocysteine geometric means were significantly higher $(p=0,005)$ in men $(9,97 \pm 4,81 \mathrm{mmol} / \mathrm{L})$ than in women $(7,35 \pm 2,03 \mathrm{mmol} / \mathrm{L})$. Plasma homocysteine level was significantly correlated with folic acid plasma level $(\mathrm{r}=-0,329, p=0,007)$ but not with B-12 vitamin. However, plasma homocysteine level was correlated with body mass index $(\mathrm{r}=0,391 ; p=0,001)$. Conclusions: The distribution of homocysteine levels in our sample is within the reference range already reported. However, there was a complete shift of the homocysteine distribution curve towards lower values. Plasma folic acid levels may be a determinant factor in the variation of homocysteine levels.

Key words: Homocysteine; folic acid; vitamin B-12; fluorescence polarization immunoassay.

\footnotetext{
Correspondencia:

Dra. María Mercedes Soberón Lozano

CIBN, Facultad de Medicina - UNMSM

Av. Grau 755. Lima 01, Perú.

E-mail: msoberonl@sanfer.unmsm.edu.pe
} 


\section{INTRODUCCIÓN}

La homocisteína es un aminoácido con un grupo sulfhidrilo susceptible a oxidación a $\mathrm{pH}$ fisiológico. En el plasma, la homocisteína se encuentra en su forma reducida, en $1 \%$ aproximadamente; cerca de $70 \%$ se encuentra unida a albúmina y, el resto (29\%), formando compuestos disulfuro de peso molecular bajo. La suma de todas estas especies de homocisteína es denominada en la literatura homocisteína total.

La homocisteína es producida por el organismo humano a partir de metionina. Cuando los niveles de metionina están elevados, la homocisteína es utilizada para la síntesis de la cisteína de manera irreversible. La primera reacción de esta vía es catalizada por una enzima dependiente de la vitamina B-6, la cistationinab-sintetasa (CBS). Si los niveles de metionina son bajos, la homocisteína es utilizada para mantener los niveles de metionina a través de varias vías de remetilación. En los tejidos extrahepáticos, la homocisteína es remetilada por acción catalítica de la enzima metionina sintasa (MS), que es dependiente de la vitamina B-12, y que utiliza al metil tetrahidrofolato como dador de grupos metilo. A su vez, la síntesis de metiltetrahidrofolato es catalizada por la enzima metilentetrahidrofolato reductasa (MTHFR), dependiente de la vitamina B-2. De tal forma que, la deficiencia de estas vitaminas o falla en la síntesis de estas enzimas, ocasionaría una elevación de homocisteína en el plasma y, por lo tanto, explicaría los casos de errores congénitos del metabolismo de la homocisteína, comúnmente denominada homocisteinuria $\left({ }^{1}\right)$.

En 1962, Gerritsen $\left({ }^{2}\right)$ y Carson $\left({ }^{3}\right)$ comunicaron la presencia de homocisteína en la orina de niños con retardo mental. Dos años más tarde, Mudd y colaboradores $\left({ }^{4}\right)$ encontraron que la enzima CBS era deficiente en estos niños y, además, que eventos tromboembólicos eran frecuentes en estos pacientes.

En 1969, McCully y col. $\left({ }^{5}\right)$, describieron la patología vascular con efectos tromboembólicos en pacientes con homocisteinuria y concluyeron que una de las causas de este tipo de trastorno vascular era la presencia de niveles altos de homocisteína.

En 1972, nuevamente Mudd y col. encontraron casos de homocisteinuria asociados a la deficiencia de la enzima MTHFR $\left(^{6}\right)$ y a defectos en el metabolismo de la vitamina B-12. Un cuadro clínico de eventos tromboembólicos similar a la deficiencia de la enzima CBS fue observado en estos pacientes.

En 1976, Wilcken y Wilcken $\left({ }^{7}\right)$ comunicaron por vez primera una patogénesis de las arterias coronarias de pacientes que implicaba un metabolismo anormal de homocisteína.

En los años 1992-93, la concurrencia de trabajos sobre homocisteína y enfermedades coronarias aumentó, lo que conllevó a desarrollar un metaanálisis de los estudios ya realizados. Es así que a partir del año 1995, se realizó los primeros estudios mostrando que la homocisteína era un factor de riesgo independiente en ateroesclerosis, enfermedades coronarias, cerebrovasculares y vasos periféricos $\left({ }^{8,9}\right)$.

El proyecto de la Acción Concertada Europea de Homocisteína y Enfermedades Vasculares ha reconocido como rangos normales de homocisteína total en adultos de 5 a $15 \mathrm{mmol} / \mathrm{L}$ (media de $10 \mathrm{mmol} / \mathrm{L}$ ). Se ha denominado hiperhomocisteinemia a valores plasmáticos de homocisteína total mayores a $15 \mathrm{mmol} / \mathrm{L}$. Esta hiperhomocisteinemia puede ser moderada (15 a $30 \mathrm{mmol} / \mathrm{L}$ ), intermedia ( 30 a $100 \mathrm{mmol} / \mathrm{L}$ ) y severa (mayores de $100 \mathrm{mmol} / \mathrm{L}$ ) $\left(^{10,11}\right)$. Sin embargo, existen marcadas diferencias en los rangos de valores referenciales encontrados en la literatura, debido a la diversidad de metodologías utilizadas, diferencias en el manejo de la muestra y, principalmente, en la diversidad de factores nutricionales y étnicos presentes en las diferentes poblaciones estudiadas.

Los estudios antes mencionados han mostrado la posibilidad de que este aminoácido sea un marcador de prevención para trastornos cardiovasculares. Por tal motivo, es importante 
hacer un estudio de la distribución de los niveles de homocisteína en nuestro país y confrontar los valores referenciales extraídos de la literatura con los de nuestra población, toda vez que los estilos de vida en nuestro país difieren de los países europeos y de Norteamérica, donde se ha obtenido estos valores. Además, debido a que el metabolismo de homocisteína está relacionado con los niveles de algunas vitaminas del complejo $\mathrm{B}$, es necesario hacer el estudio relacionando estos metabolitos.

\section{MATERIALES Y MÉTODOS}

La muestra seleccionada aleatoriamente fue de 65 jóvenes con edades comprendidas entre 18 a 30 años, alumnos de las Facultades de Medicina Humana y Ciencias Matemáticas de la Universidad Nacional Mayor de San Marcos. Se excluyó sujetos con trastornos cardiovasculares, diabetes y antecedentes familiares de esta índole. Los sujetos seleccionados firmaron un documento de consentimiento informado donde se les explicó el propósito del estudio, el procedimiento a seguir, las inconveniencias y riesgos a los que se someterían, los beneficios que recibirían al participar y la confidencialidad del trabajo. Adicionalmente, se elaboró una ficha con los datos personales, incluyendo talla, peso, género, presencia de enfermedades y antecedentes familiares. Finalmente, se aplicó una encuesta sobre frecuencia de consumo de alimentos ricos en ácido fólico y vitamina B-12.

Las muestras de plasma sanguíneo fueron recolectadas en frascos vacutainer con anticoagulante EDTA $\mathrm{K}_{3}$, sometidas inmediatamente a centrifugación a $1300 \mathrm{x}$ g, por 15 minutos, y almacenadas a $-20{ }^{\circ} \mathrm{C}$ hasta su respectivo análisis.

El dosaje de homocisteína plasmática fue por inmunoensayo de polarización fluorescente (FPIA), proporcionado por los Laboratorios Abbott SA, fundamentado en un proceso de reducción, conversión enzimática e inmunoensayo $\left({ }^{12}\right)$.
El dosaje de ácido fólico fue por captura iónica, IMx Folato, de los Laboratorios Abbott SA, basado en la formación de complejos polianión-analito con carga negativa, los que son capturados a través de una interacción electrostática con la matriz, que presenta una carga eléctrica positiva $\left({ }^{13}\right)$.

Se determinó la vitamina B-12 por enzimoinmunoensayo de micropartículas, del laboratorio antes mencionado; es un ensayo enzimático de micropartículas recubiertas de un factor intrínseco para la vitamina B-12 $\left({ }^{14}\right)$.

Las variables estudiadas fueron operacionalizadas según los valores referenciales que se describe a continuación: para vitamina $\mathrm{B}-12$, en el rango de 223 a $1132 \mathrm{pg} / \mathrm{mL}$; para folato de 3,5 a $16,1 \mathrm{ng} / \mathrm{mL}$; y para homocisteína de 5 a $15 \mathrm{mmol} / \mathrm{L}$.

Se obtuvo las distribuciones percentiles de los niveles de homocisteína, ácido fólico y vitamina B-12. La valoración de la relación entre estas variables se realizó mediante el coeficiente de correlación de Pearson.

\section{RESULTADOS}

Se evaluó una muestra de 65 jóvenes de ambos sexos, con una edad promedio de $20,7 \pm 3,2$ años; la proporción de varones y mujeres fue aproximadamente igual $(54,4 \%$ y $44,6 \%$, respectivamente). El 75,4\% presentó índices de masa corporal (IMC) dentro de los rangos normales (de 18 a 25) (Tabla 1).

Tabla 1.- Distribución de los sujetos de acuerdo al sexo e IMC.

\begin{tabular}{lccc}
\hline \multirow{2}{*}{ IMC } & \multicolumn{2}{c}{ Sexo } & \multirow{2}{*}{ Total } \\
\cline { 2 - 3 } & $\begin{array}{c}\text { Varones } \\
\%\end{array}$ & $\begin{array}{c}\text { Mujeres } \\
\%\end{array}$ & $\%$ \\
\hline Normal $(<25)$ & 66,7 & 86,2 & 75,4 \\
Sobrepeso (25-29) & 22,2 & 13,8 & 18,5 \\
Obesidad $(\geq 30)$ & 11,1 & & 6,2 \\
\hline
\end{tabular}


La distribución de los niveles de homocisteína (Figura 1) varió entre 1,4 y 24,04 $\mathrm{mmol} / \mathrm{L}$. Considerando los rangos de normalidad publicados en la literatura (5-15 $\mathrm{mmol} / \mathrm{L})$, se observa que $85,9 \%$ de los sujetos estudiados está dentro de ese rango, con una mediana de 7,76 $\mathrm{mmol} / \mathrm{L}$. Por otro lado, considerando un nivel de confianza del 95\%; el nivel medio de homocisteína plasmática fluctuó entre 7,80 y $9,80 \mathrm{mmol} / \mathrm{L}$. Las medias de homocisteína para varones y mujeres fueron $9,97 \pm 4,81 \mathrm{mmol} / \mathrm{L}$ y $7,35 \pm 2,03 \mathrm{mmol} / \mathrm{L}$, respectivamente. La prueba $\mathrm{T}$ para muestras independientes señaló diferencias significativas $(p=0,005)$ en los niveles de homocisteína para ambos sexos.

La distribución de las concentraciones para vitamina B-12 varió entre 128,2 y 795,1 pg/mL (Figura 2) y, para ácido fólico, entre 2,0 y 13,4 ng/mL (Figura 3). Un porcentaje no despreciable de los jóvenes presentó niveles de vitamina B-

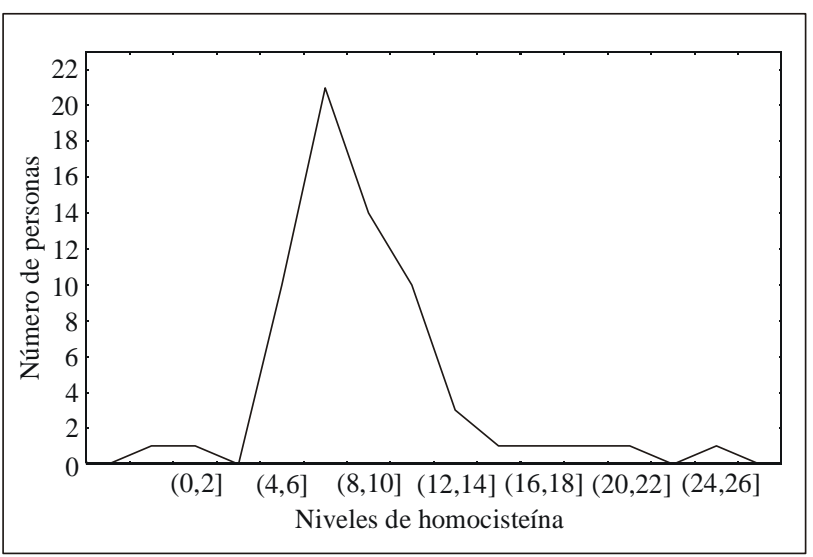

\begin{tabular}{cc}
\hline $\begin{array}{c}\text { Niveles de homocisteína } \\
(\mathrm{mmol} / \mathrm{L})\end{array}$ & $\begin{array}{c}\text { Porcentaje } \\
(\%)\end{array}$ \\
\hline$<5,0$ & 6,3 \\
$5,0-9,9$ & 65,6 \\
$10,0-15,0$ & 20,3 \\
$>15,0$ & 7,8 \\
Total & 100,0 \\
\hline
\end{tabular}

Figura 1.- Distribución porcentual de los niveles de homocisteína en relación a valores referenciales internacionales.
12 y ácido fólico por debajo de los rangos de normalidad (22,2\% y 9,2\%, respectivamente). Las medias fueron $5,19 \pm 2,06 \mathrm{ng} / \mathrm{mL}$ para ácido fólico y $314,95 \pm 127,28 \mathrm{pg} / \mathrm{mL}$ para vitamina B-12, las medianas de $4,70 \mathrm{ng} / \mathrm{mL}$ y $294,60 \mathrm{pg} /$ $\mathrm{mL}$, respectivamente, lo que indica que la distribución de estas vitaminas en la población estudiada está sesgada hacia niveles bajos.

En la Figura 4 se observa el diagrama de dispersión relacionando los niveles de homocisteína y ácido fólico. El coeficiente de correlación de Pearson $(\mathrm{r}=-0,329)$ resultó estadísticamente significativo $(p=0,007)$, pero no se encontró evidencias de asociación entre los niveles de homocisteína y vitamina B-12 .

Asimismo, se encontró asociación entre los niveles de homocisteína e índice de masa corporal (Figura 5). El coeficiente de correlación de Pearson $(r=0,391)$ resultó estadísticamente significativo $(p=0,001)$.

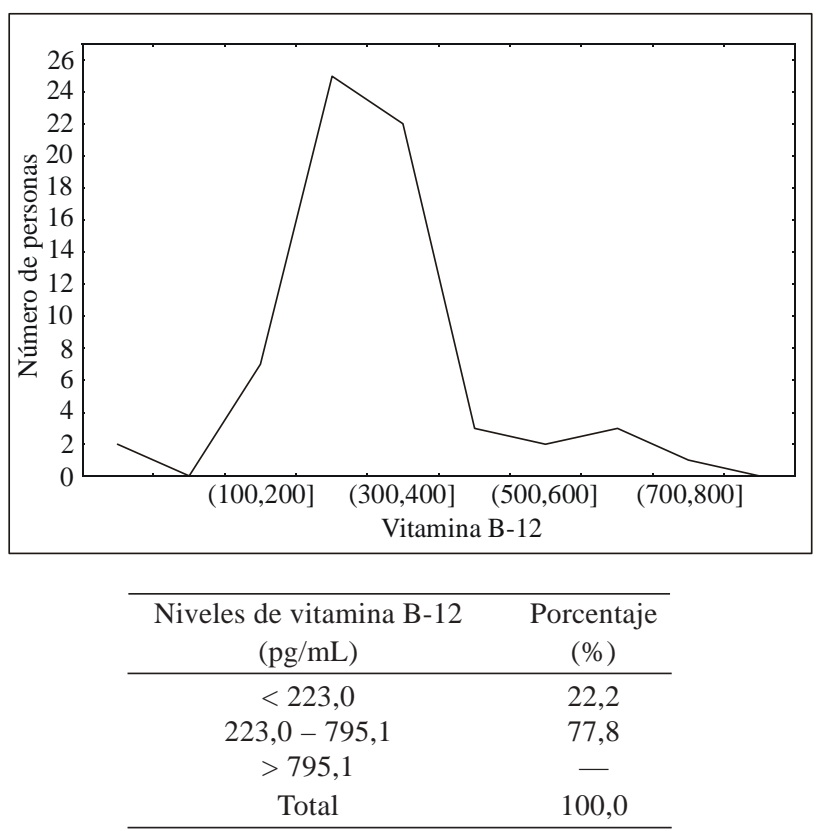

Figura 2.- Distribución porcentual de vitamina B-12 en relación a valores referenciales internacionales. 


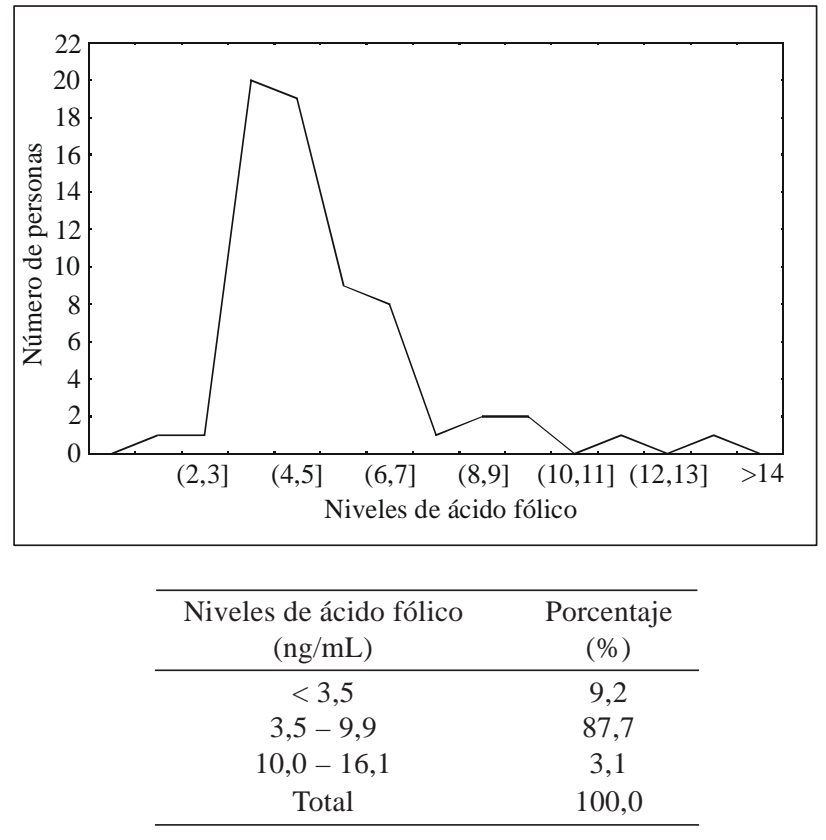

Figura 3.- Distribución porcentual de ácido fólico en relación a valores referenciales internacionales.

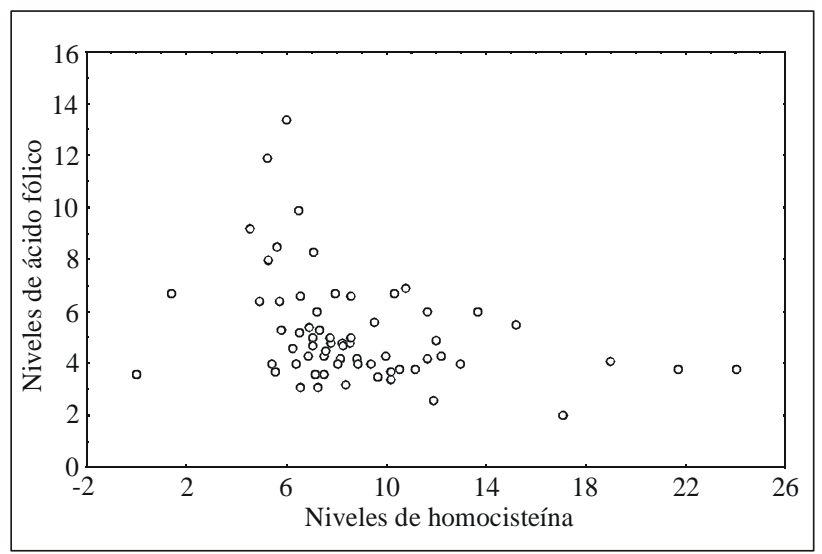

Figura 4.- Diagrama de dispersión de los niveles de homocisteína y ácido fólico.

En la encuesta de frecuencia de consumo de alimentos, se colocó los alimentos representativos de estas vitaminas; por ejemplo, para la vitamina B-12 fueron principalmente las carnes rojas, para el ácido fólico los vegetales de hojas verdes. Los alimentos incluidos fueron, en la medida de lo posible, aquellos que nuestra población frecuentemente conoce y consume. Al evaluar los hábitos de consumo de alimentos, se encontró que los jóvenes se caracterizaron por un bajo consumo de alimentos ricos en ácido fólico y vitamina B-12 (Figura 6), observándose además que la mayoría de estos alimentos son consumidos con una frecuencia semanal o mensual (Tabla 2).

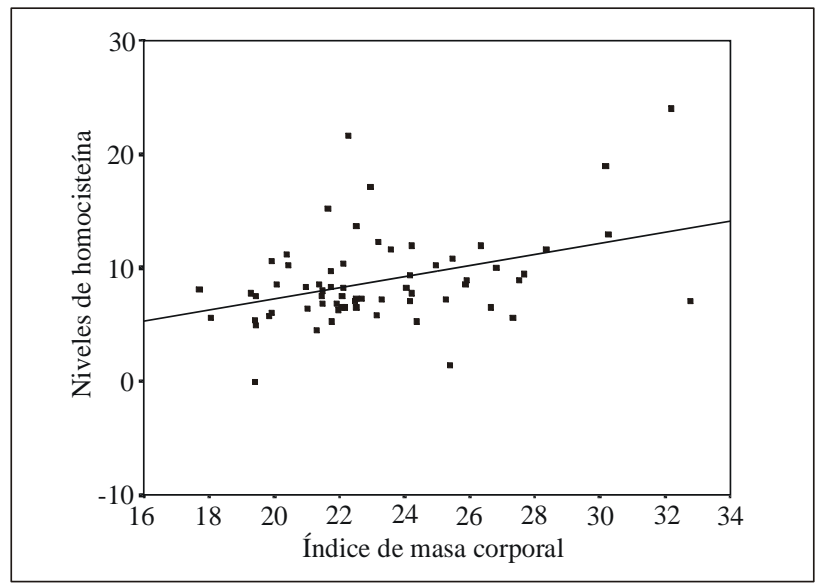

Figura 5.- Diagrama de dispersión de los niveles de homocisteína e índice de masa corporal.

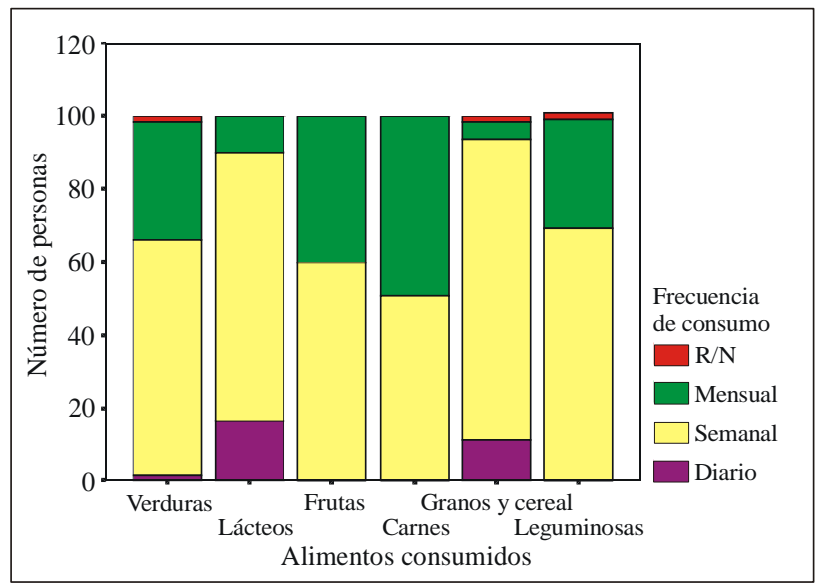

Figura 6.- Frecuencia de consumo de alimentos ricos en ácido fólico y vitamina B-12. 
Tabla 2.- Frecuencia de consumo de alimentos ricos en ácido fólico y vitamina B-12.

\begin{tabular}{lcccc}
\hline \multirow{2}{*}{ Alimentos } & \multicolumn{5}{c}{ Frecuencia de consumo } \\
\cline { 2 - 6 } & $\begin{array}{c}\text { Diario } \\
\%\end{array}$ & $\begin{array}{c}\text { Semanal } \\
\%\end{array}$ & $\begin{array}{c}\text { Mensual } \\
\%\end{array}$ & $\begin{array}{c}\text { R/N* } \\
\%\end{array}$ \\
\hline Verduras & 1,6 & 64,5 & 32,3 & 1,6 \\
Lácteos & 15,0 & 76,0 & 9,0 & - \\
Frutas & - & 59.7 & 40.3 & - \\
Granos y cereales & 11,3 & 82,3 & 4,8 & 1,6 \\
Carnes, vísceras, huevos & - & 50,8 & 49,2 & - \\
Leguminosas & - & 69,4 & 29,9 & 1,6 \\
\hline
\end{tabular}

$* R / N=$ Rara vez o nunca.

\section{DISCUSIÓN}

Se realizó un estudio exploratorio y de corte transversal, en una población aparentemente saludable de sujetos residentes en la ciudad de Lima, Perú, con un rango de edades de 18 a 30 años, estudiantes de la Universidad Nacional Mayor de San Marcos. La mayor parte de los sujetos presentó valores de índice de masa corporal dentro de los rangos normales (Tabla 1). La necesidad de escoger un rango de edades estrecho es importante, ya que se observa que los valores de homocisteína varían notablemente con la edad; por ejemplo, en niños estos valores son aproximadamente la mitad de los que se estima en la literatura como valores referenciales $\left({ }^{15}\right) \mathrm{y}$, en general, existe una tendencia de incremento en los niveles de homocisteína en individuos mayores de 40 años $\left({ }^{16,17}\right)$.

La literatura reporta el rango de 5 a $15 \mathrm{mmol} /$ L como valores referenciales para homocisteína en adultos, con una media de $10 \mathrm{mmol} / \mathrm{L}$ ${ }^{(11)}$. Teniendo en cuenta estos datos, se pudo observar que los niveles de homocisteína plasmática en la población estudiada (Figura 1) estuvieron dentro de estos valores referenciales; sin embargo, $71,9 \%$ de ellos tiene niveles menores de $10 \mathrm{mmol} / \mathrm{L}$, con una media de $8,32 \pm 5,46 \mathrm{mmol} / \mathrm{L}$. Probablemente, el valor medio de homocisteína encontrado en la población de estudio, difiere de la media internacional debido al rango de edades seleccionado y a las condiciones nutricionales que presentó la muestra. Además, los valores de homocisteína fueron significativamente mayores en varones $(9,97 \pm 4,81 \mathrm{mmol} / \mathrm{L})$, que en mujeres $(7,35 \pm 2,03 \mathrm{mmol} / \mathrm{L})$. Esto se evidencia al comparar nuestros resultados con los obtenidos por Nygard y colaboradores (18), quienes demuestran que en sujetos noruegos con edades entre 40 y 42 años, el valor medio fue $10 \mathrm{mmol} / \mathrm{L}$ en varones y $9,1 \mathrm{mmol} / \mathrm{L}$ en mujeres; y, para las edades de 65 a 67 años, de $12,3 \mathrm{mmol} /$ $\mathrm{L}$ en varones y $11,0 \mathrm{mmol} / \mathrm{L}$ en mujeres. Resultados similares fueron obtenidos por los mismos autores en sujetos holandeses $\left({ }^{19}\right)$.

Estudios previos sugieren tomar en consideración los niveles de las vitaminas que participan en el metabolismo de la homocisteína $\left.{ }^{(1,20}\right)$. Por tal motivo, se examinó la distribución de las vitaminas ácido fólico y B-12 en la población seleccionada, a través de muestras sanguíneas y, además, de una ficha de frecuencia de consumo de alimentos. Los resultados obtenidos en las Figuras 2 y 3 muestran que la población en estudio presenta niveles plasmáticos de ácido fólico y vitamina B-12 dentro de los rangos establecidos por la literatura, pero, con tendencia a los límites inferiores; esto está relacionado con la frecuencia de consumo de alimentos, la cual reporta también una baja ingesta de vitamina B-12 y de ácido fólico. Los resultados indican que no se estaría cubriendo los requerimientos para un adecuado metabolismo de homocisteína. Sin embargo, nuestra población no presenta niveles elevados de homocisteína como una consecuencia de los niveles bajos de las vitaminas ácido fólico y B12 , lo cual llama nuestra atención, ya que trabajos reportados por la literatura han mostrado que una hiperhomocisteinemia moderada puede ser atribuida a la deficiencia de las vitaminas ácido fólico y B-12; esta alteración se ha logrado revertir con una adecuada ingesta de estas vitaminas $\left({ }^{21-25}\right)$. El análisis estadístico de nuestros datos muestra una correlación significativa entre los niveles de homocisteína y ácido fólico, pero no se ha encontrado asociación 
entre los niveles de homocisteína y vitamina B12 , como se muestra en otros estudios $\left({ }^{1,20}\right)$.

Nuestro más importante hallazgo, dada la relación de la homocisteína con las vitaminas B-12 y ácido fólico, es sin duda el estado nutricional de la población en estudio, que refleja la realidad de una gran mayoría de habitantes peruanos, es decir, niveles bajos de vitaminas del complejo B y, a la vez, valores de homocisteína sesgados hacia niveles inferiores, producto de una probable deficiencia en el consumo de proteínas, tal como se observó en la encuesta de frecuencia de consumo de alimentos. Esta realidad no se aparta de otros países del continente americano en vías de desarrollo, como por ejemplo, Costa Rica, que reporta una media de los niveles de homocisteína plasmática en una población urbana de $8,9 \mathrm{mmol} /$ $\mathrm{L}$ en varones y 7,3 mmol/L en mujeres; y para ácido fólico, valores menores de 6,8 $\mathrm{mmol} / \mathrm{L}$ $\left.{ }^{26}\right)$. Sin duda, la diversidad étnica y los hábitos nutricionales marcan una diferencia notable en la distribución de la homocisteína comparada con la de los países europeos y de Norteamérica, de donde se ha obtenido los valores referenciales. Por otro lado, no se descarta la idea de una probable variedad polimórfica de los genes en el hombre peruano, que controla la síntesis de las enzimas MS y MTHFR, las que son dependientes de las vitaminas estudiadas, aspecto que sería interesante investigar $\left({ }^{27}\right)$.

La encuesta de frecuencia de consumo de alimentos mostró un consumo mayormente semanal y hasta mensual y escasamente diario de los productos ricos en ácido fólico y vitamina B-12, lo que se corrobora con los datos obtenidos en las muestras sanguíneas, es decir, niveles bajos de las vitaminas ácido fólico y B-12.

En Norteamérica, además de la determinación de los factores de riesgo clásicos de trastornos cardiovasculares, se hace ya un dosaje de homocisteína plasmática en pacientes que presentan estas alteraciones; los valores obtenidos de este aminoácido en la población de estudio podrían ser tomados en cuenta como un dato referencial para la prevención de este tipo de enfermedades $\left({ }^{10,28-30}\right)$.

Se concluye que la media poblacional de los niveles de homocisteína plasmática en la muestra seleccionada fue de $8,32 \pm 5,46 \mathrm{mmol} / \mathrm{L}$. Los niveles de ácido fólico y vitamina B-12 presentan una distribución sesgada hacia valores bajos (ver Figuras 1, 2 y 3), Existe una correlación significativa entre los niveles de homocisteína y ácido fólico, pero no hay evidencia de asociación entre los niveles de homocisteína y vitamina B12, por lo que los niveles de ácido fólico en sangre pueden ser un factor determinante de la variación de los niveles de homocisteína en la población estudiada.

Estas conclusiones permiten recomendar a las instituciones pertinentes y autoridades correspondientes la ejecución de programas de orientación y políticas nutricionales que ayuden al estudiante sanmarquino $\mathrm{y}$, en general, a la población peruana, a tener hábitos alimenticios adecuados para ingerir la diversidad de alimentos cultivados en nuestro país que nos proporcionan macro y micronutrientes necesarios para una buena salud; y, asimismo, establecer programas de prevención que eviten el incremento de padecimientos cardiovasculares en nuestra población.

\section{Agradecimientos}

Agradecemos al Dr. Sergio Gerardo Ronceros Medrano, así como, a los representantes de Atlanta S.A. Lic.T.M. Violeta Carbajal Goudey y Lic. T.M. Elard Salinas Córdova, por sus valiosas colaboraciones en la realización de este proyecto. También, agradecemos al Consejo Superior de Investigaciones de la Universidad Nacional Mayor de San Marcos por el apoyo financiero proporcionado al proyecto (Estudio $\mathrm{N}^{\circ} 020104201$ ).

\section{REFERENCIAS BIBLIOGRÁFICAS}

1. Selhub J. Homocysteine metabolism. Annu Rev Nutr 1999;19:217-46. 
2. Gerritsen T, Vaughn JG, Weisman HA. The identification of homocysteine in the urine. Biochem Biophys Res Commun 1962;9:493.

3. Carson NAJ, Neill DW. Metabolic abnormalities detected in a survey of mentally backward individuals in Northern Ireland. Arch Dis Child 1962;37:505-13.

4. Mudd SH, Finkelstein JD, Irreverre F, Laster L. Homocystinuria: an enzymatic defect. Science 1964;143:1443-5.

5. McCully KS. Vascular pathology of homocysteinemia: implications for the pathogenesis of arteriosclerosis. Am J Pathol 1969;56:111-28.

6. Mudd SH, Uhlendorf BW, Freeman JM, Finkelstein JD, Shih VE. Homocystinuria associated with decreased methylenetetrahydrofolate reductase activity. Biochem Biophys Res Commun 1972;46:905-12.

7. Wilken DEL, Wilcken B. The pathogenesis of coronary artery disease. A possible role for methionine metabolism. J Clin Invest 1976;57:1079-82.

8. Boushey CJ, Beresford SA, Omenn GS, Motulsky AG. A quantitative assessment of plasma homocysteine as a risk factor for vascular disease: probable benefits of increasing folic acid intakes. JAMA 1995;274:1049-57.

9. Stubbs PJ. Effect of plasma homocysteine concentration on early and late events in patients with acute coronary syndromes. Circulation 2000;102:605-10.

10. Graham IM, Daly LE, Refsum HM, Robinson K, Brattstrom LE, Ueland PM, et al. Plasma homocysteine as a risk factor for vascular disease: The European Concerted Action Project. JAMA 1997;277:1775-81.

11. Ueland PM, Refsum H, Stabler SP, Malinow MR, Andersson A, Allen RH. Total homocysteine in plasma or serum. Methods and clinical applications. Clin Chem 1993;39:1764-79.

12. Abbott Diagnostics Division. IMx System Homocysteine. Mexico DF: Abbott Laboratories; 2001.

13. Abbott Diagnostics Division. IMx System Folate. Illinois: Abbott Laboratories; 2001.

14. Abbott Diagnostics Division. IMx System Vitamin B-12. Wiesbaden: Abbott Laboratories; 1997.

15. Osganian SK, Stampfer MJ, Spiegelman D, Rimm E, Cutler JA, Feldman HA, et al. Distribution of and factors associated with serum homocysteine levels in children: child and adolescent trial for cardiovascular health. JAMA 1999;281:1189-96.

16. Lussier-Cacan S, Xhignesse M, Piolot A, Selhub J, Davignon J, Genest J Jr. Plasma total homocysteine in healthy subjects: sex-specific relation with biological traits. Am J Clin Nutr 1996;64:587-93.

17. Andersson A, Brattstrom L, Israelsson B, Isaksson A, Hamfelt A, Hultberg B. Plasma homocysteine before and after methionine loading with regard to age, gender and menopausal status. Eur J Clin Invest 1992;22:79-87.

18. Nygård O, Vollset SE, Refsum H, Stensvold I, Tverdal A, Nordrehaug JE, et al. Total plasma homocysteine and cardiovascular risk profile. The Hordaland Homocysteine Study. JAMA 1995;274:1526-33.

19. Nygard O, Refsum H, Ueland PM, Vollset SE. Major lifestyle determinants of plasma total homocysteine distribution: the Hordaland Homocysteine Study. Am J Clin Nutr 1998;67:263-70.

20. Refsum H, Ueland P, Nygard O, Vollset SE. Homocysteine and cardiovascular disease. Annu Rev Medicine 1998;49:3162.

21. Naurath HJ, Joosten E, Rieszler R, Stabler SP, Allen RH, Lindenbaum J. Effects of vitamin B12, folate, and vitamin B6 supplements in elderly people with normal serum vitamin concentrations. Lancet 1995;346:85.

22. Franken DG, Boers GHJ, Blom HJ, Trijbels FJM, Kloppenborg PW. Treatment of mild hyperhomocysteinemia in vascular disease patients. Arterioscl Thromb 1994;14:465.

23. Brattstrom L, Lindgren A, Israelsson B, Anderson A, Hultberg B. Homocysteine and cysteine: Determinants of plasma levels in middle-aged and elderly subjects. J Intern Med 1994;236:631.

24. Herrmann W, Obeid R, Jouma M. Hyperhomocysteinemia and vitamin B-12 deficiency are more striking in Syrians than in Germans: causes and implications. Atherosclerosis 2003;166(1):143-50.

25. Oltean S, Banerjee R. Nutritional modulation of gene expression and homocysteine utilization by vitamin B-12. J Biol Chem 2003;278(23):20778-84.

26. Kim MK, Ordovas JM, Selhub J, Campos H. B vitamins and plasma homocysteine concentrations in an urban and rural area of Costa Rica. J Am College Nut 2003;22:22431.

27. Jacques PF, Bostom AG, Selhub J, Rich S, Ellison RC, Eckfeldt JH, et al. Effects of polimorphisms of methionine synthase reductase on total plasma homocysteine in the NHLBI Family Heart Study. Atherosclerosis 2003;166(1):49-55.

28. Ueland PM, Refsum H, Beresford SAA, Vollset SE. The controversy over homocysteine and cardiovascular risk. Amer J Clin Nutr 2000;72:324-32.

29. Guilland JC, Favier A, Potier de Courcy G, Galan P, Hercberg S. Hyperhomocysteinemia: an independent risk factor or a simple marker of vascular disease? 1. Pathol Biol 2003;51(2):101-10.

30. Guilland JC, Favier A, Potier de Courcy G, Galan P, Hercberg S. Hyperhomocysteinemia: an independent risk factor or a simple marker of vascular disease? 2. Pathol Biol 2003;51(2):111-21. 